\title{
Current Concepts on Dedifferentiation/High-Grade Transformation in Salivary Gland Tumors
}

\author{
Ana Flávia Costa, ${ }^{1}$ Albina Altemani, ${ }^{1}$ and Mario Hermsen ${ }^{2}$ \\ ${ }^{1}$ Department of Pathology, University of Campinas, (UNICAMP), 13083-887 Campinas, SP, Brazil \\ ${ }^{2}$ Department of Otolaryngology, IUOPA, Hospital Universitario Central de Asturias, 33006 Oviedo, Spain
}

Correspondence should be addressed to Ana Flávia Costa, costa.anaflavia@gmail.com

Received 15 November 2010; Accepted 9 May 2011

Academic Editor: Stefan Pambuccian

Copyright (C) 2011 Ana Flávia Costa et al. This is an open access article distributed under the Creative Commons Attribution License, which permits unrestricted use, distribution, and reproduction in any medium, provided the original work is properly cited.

\begin{abstract}
The concept of dedifferentiation had previously been used in salivary gland carcinomas. Recently, the term "high-grade transformation" was introduced for adenoid cystic carcinoma, acinic cell carcinoma, epithelial-myoepithelial carcinoma, and polymorphous low-grade adenocarcinoma and may better reflect this phenomenon, although transformation into moderately differentiated adenocarcinoma (i.e., not "high grade") has also been described. Among the immunohistochemical markers, Ki67 seems to be the only one that can help distinguish between the conventional and transformed components; however, the combination of morphological criteria is still sovereign. The overexpression of p53 was observed in the transformed component in all tumor types studied, despite few cases having been demonstrated to carry mutations or deletions in TP53 gene. Genetic studies in salivary gland tumors with dedifferentiation/high-grade transformation are rare and deserve further investigation. This paper aims at providing an overview on the recent concepts in histopathological classification of salivary gland tumors, complemented by immunohistochemical and genetic findings.
\end{abstract}

\section{Introduction}

The concept of dedifferentiation was first proposed by Dahlin and Beabout in 1971 [1], when they described dedifferentiated chondrosarcoma as a distinct clinicopathologic entity characterized by a low-grade chondrosarcoma juxtaposed to a histologically different high-grade sarcoma [1]. Lately, dedifferentiation has been recognized in a variety of salivary gland carcinomas, including adenoid cystic carcinoma [2], mucoepidermoid carcinoma [3], myoepithelial carcinoma [4], epithelial-myoepithelial carcinoma [5], and acinic cell carcinoma [6].

Dedifferentiation is the progression of cells towards a less differentiated state in which the original line of differentiation is no longer evident [7]. The term dedifferentiation might not be properly used in epithelial tumors, especially when the dedifferentiated component is still recognizable as carcinoma or adenocarcinoma [8]. Recently, Seethala et al. introduced the term "high-grade transformation" for adenoid cystic carcinomas. This term better reflects the fact that the dedifferentiated component often maintains some features of the original tumor, such as glandular differentiation [8-10]. In recent studies, our group and others have demonstrated that adenoid cystic carcinomas can also undergo transformation to adenocarcinomas which are not poorly differentiated, suggesting that also the term "highgrade transformation" may not be adequate, at least in the case of adenoid cystic carcinoma [11, 12].

Although considerable progress has been made in elucidating the genetic events that underlie the progression of many malignancies, those involved in salivary gland tumors are still poorly understood and the relationship between histological progression and genetic events is not well defined. The general theory of monoclonal evolution assumes that the mutational complexity of a tumor increases with time and, therefore, tumor genomes with the fewest chromosome aberrations contained the earliest mutations in tumor progression $[13,14]$. In contrast, high-resolution comparative genomic hybridization (CGH) microarrays have been used to study the genome structure of heterogeneous breast 
tumors and shown that they progress by different genomic rearrangement patterns [15]. Thus, the genomic heterogeneity can be ascribed to genetically distinct subpopulations, which contain a set of common mutations (early events) that are inherited and persistent throughout their evolution, while events unique to the profiles are late [16]. However, genetic alterations cannot solely explain the histological heterogeneity in tumors. Epigenetic alterations, such as DNA hypomethylation in tumor cells, cause chromatin decondensation and chromosomal rearrangements that may result in chromosomal instability. Moreover, DNA hypermethylation of $\mathrm{CpG}$ islands near the promoter regions silences specific genes including tumor suppressor genes in cooperation with histone modification [17]. Therefore, the histological heterogeneity could also involve modifications of epigenetic switch.

This paper will give an overview on the recent concepts in histopathological classification of salivary gland tumors in which dedifferentiation/transformation has been described: adenoid cystic carcinoma, acinic cell carcinoma, epithelialmyoepithelial carcinoma, polymorphous low-grade adenocarcinoma, mucoepidermoid carcinoma, and myoepithelial carcinoma. Below follows a one-by-one description of these tumors, and emphasis will be placed on immunohistochemical and genetic findings.

\section{Clinical and Morphological Features}

Since dedifferentiation/transformation is extremely rare, there are few data to establish how the prognosis compares to that of their conventional counterparts. Several clinical features have proven to be relevant, such as lymph node metastasis in adenoid cystic carcinoma and acinic cell carcinoma $[8,9]$, recurrence in polymorphous low-grade adenocarcinoma and mucoepidermoid carcinoma [3, 18], and metastasis in epithelial-myoepithelial carcinoma [10].

The majority of adenoid cystic carcinoma (AdCC) with transformation occurs during the sixth decade or later and most commonly involves the sinonasal mucoserous glands, palate, and submandibular glands. This tumor shows a slight male predominance, unlike conventional AdCC, and is often detected at an advanced stage due to extraglandular or bone involvement. One of the most important clinical features is the high propensity for lymph node metastasis ( $57 \%$ versus $5-25 \%$ in conventional AdCC), suggesting that this tumor should be also placed in the high-risk category for neck dissection $[8,19]$. Until the time of writing, the literature revealed a total of 36 cases [2, 11, 12, 20-28]. The median survival of the largest reported series of AdCC with transformation, in which all cases were poorly differentiated carcinomas, was estimated at 12 months [8]. However, AdCC with transformation into moderately differentiated adenocarcinoma seems to present a slower course (in some cases comparable to conventional AdCC), in contrast to AdCC with transformation into poorly differentiated carcinoma which usually shows a more aggressive clinical course [11, 12]. Seethala et al. were the first to establish morphological criteria for differentiating AdCC with high-grade transformation [8]. At least three major criteria are required, proliferation of tumor cells with at least a focal loss of myoepithelial cells surrounding tumor nests, nuclear size at least 2-3 times the size of tubular/cribriform AdCC nuclei, thickened irregular nuclear membranes, and prominent nucleoli in a majority of cells. The squamous areas, micropapillary, and the loss of myoepithelial differentiation are considered unique morphological findings in the area transformed [8]. Based on the degree of gland formation (differentiation), cellular pleomorphism, and mitotic activity, Bonfitto et al. and Costa et al. classified the transformed components into moderately differentiated adenocarcinomas (when at least $2 / 3$ of the transformed component presented gland formation) and poorly differentiated carcinomas (gland formation was scarce or absent) $[11,12]$. These morphological features are observed in Figure 1. The literature does not suggest a minimum percentage of the transformed component [8].

The first acinic cell carcinoma (AcCC) with dedifferentiation/high-grade transformation of salivary gland was reported by Stanley et al., in 1988 [6]. Thirty-five cases have been described in the literature $[6,9,29-41]$ and most of them showed poor clinical outcome, but in 3 cases it was described to remain unclear [29, 42, 43]. All cases reported to date were of parotid gland origin with involvement of both the superficial or deep lobes. Especially those AcCCs in the deep parotid lobe have been associated with a poor clinical outcome [35]. The median age of 58 years is higher than that reported for conventional AcCC, 44 years [34]. In contrast with its conventional counterpart, AcCC dedifferentiation/high-grade transformation shows a slight male predominance, high recurrence rate, and high propensity for cervical lymph node metastasis, suggesting a role for neck dissection in management of patients $[9,35]$.

Dedifferentiated AcCC (Figure 2) generally shows conventional low-grade AcCC juxtaposed with high-grade carcinoma, which may be either poorly differentiated adenocarcinoma or undifferentiated carcinoma. Both solid and microcystic patterns of AcCC have been described in the low-grade component. The high-grade component generally shows a population of anaplastic cells with abundant cytoplasm, large polymorphic nuclei, and loss of acinar differentiation. Furthermore, comedonecrosis and vascular and perineural invasion are typically observed in AcCC $[6,9,35,36,38]$.

Epithelial-myoepithelial carcinoma (EMC) is a biphasic tubular neoplasm of clear myoepithelial cells surrounding small lumina lined by ductal epithelial cells. Typically, this is a low-grade malignancy that mainly occurs in the parotid gland and exhibits distinctive subtypes including tubular, papillary, cystic, and solid patterns [44]. In EMC, myoepithelial as well as ductal epithelial cells can transform into a highgrade carcinomatous component [10] which has gone under a variety of terminologies such as high-grade carcinoma $[45,46]$, dedifferentiated [5, 47-50], myoepithelial anaplasia [48], and myoepithelial carcinoma arising in EMC [2]. Recently the term "EMC with high-grade transformation" has been proposed for the all lesions where a more aggressive carcinoma is observed regardless whether it originated as a gradual transition or an abrupt transformation of the ductal or myoepithelial component [10]. The reasons for the adoption of this term were the difficulties in defining criteria for the cellular classification of the high-grade component, 

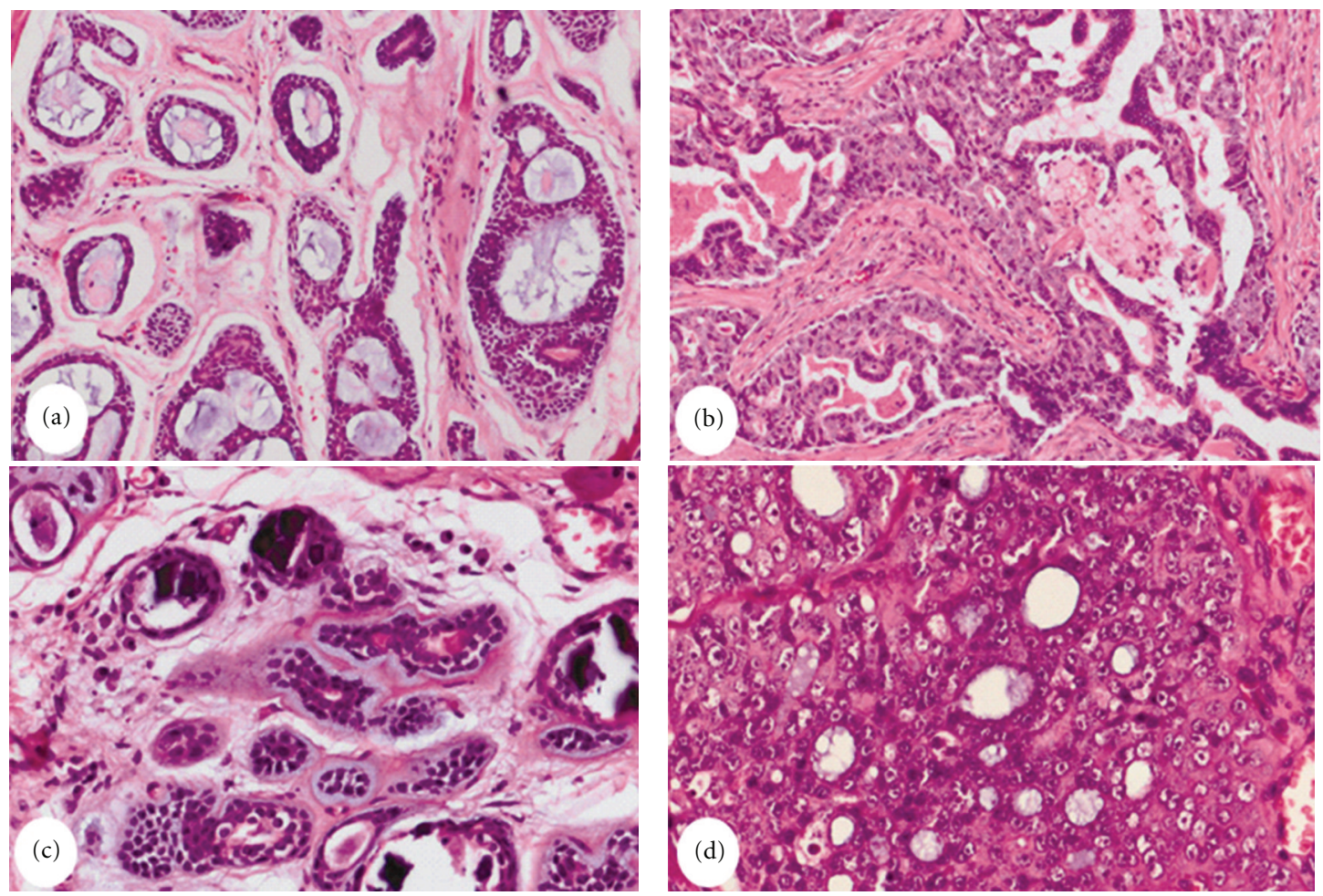

FiguRe 1: Adenoid cystic carcinoma with transformation to a moderately differentiated adenocarcinoma (a) and (b) and to poorly differentiated carcinoma (c) and (d). (a) and (b) H\&E original magnification 200x and (c) and (d), 400x.
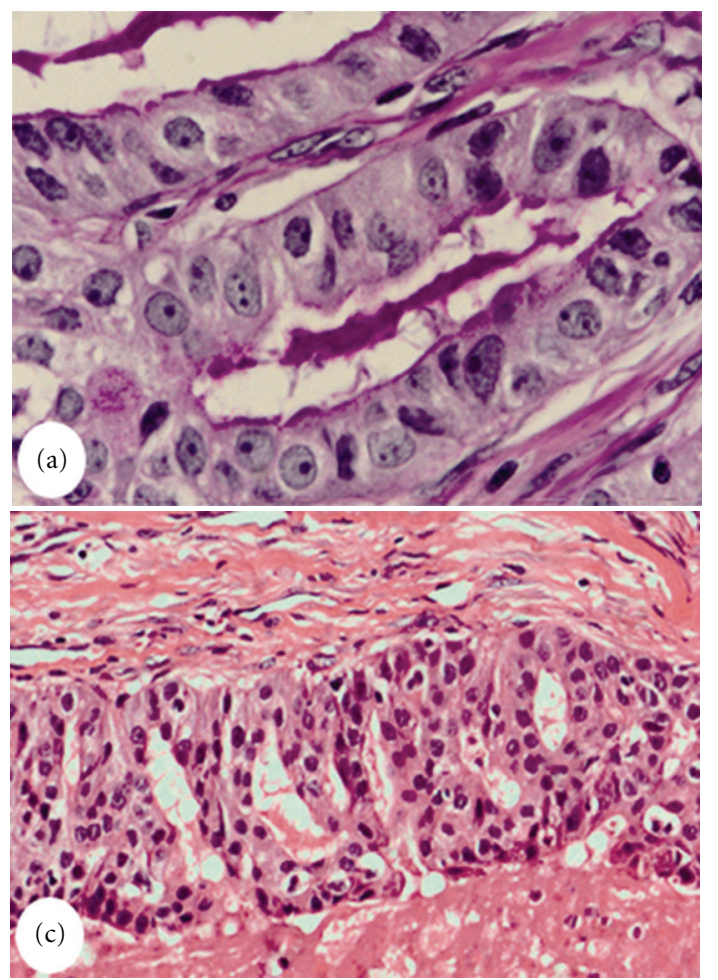

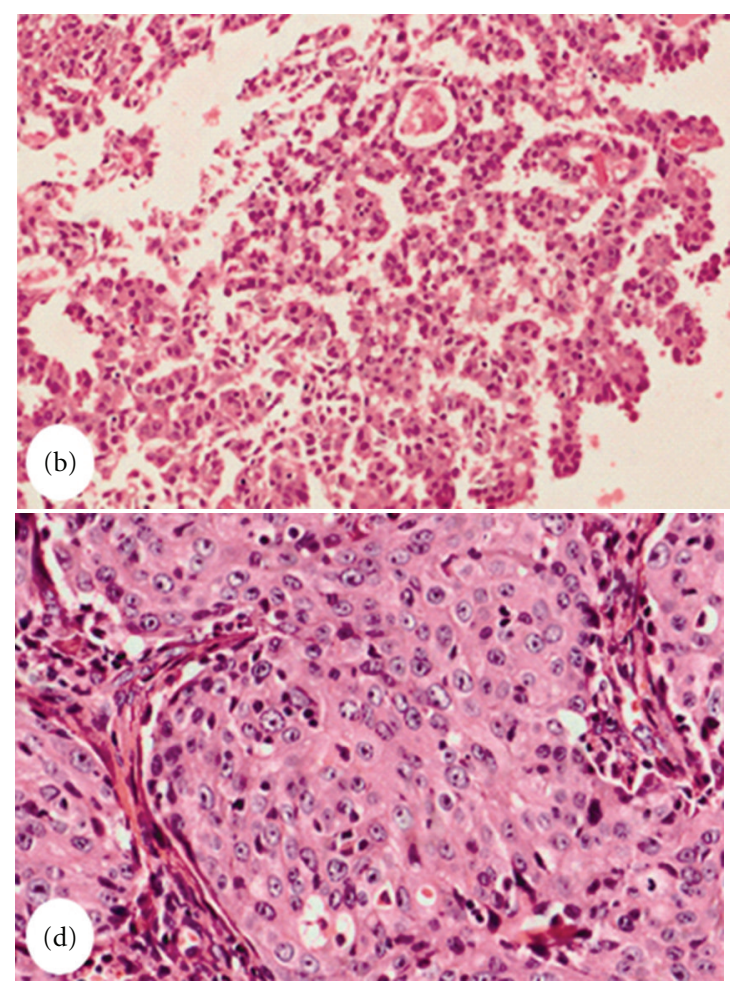

FIGURE 2: Acinic cell carcinoma with high-grade transformation to undifferentiated carcinoma. (a) and (b); (c) Conventional component; (d) high-grade transformed component. (a) PAS original magnification 1000x; (b) H\&E original magnification 100x; (c) and (d), H\&E original magnification 400x. 
the possibility that some tumors may have features of both cell types, and the fact that these lesions uniformly show worse prognosis than typical EMC.

A common feature in many of transformed EMC also is a history of indolent growth prior to the development of transformation [50]. Patients with EMC containing highgrade transformation were older than conventional EMC patients (mean 75.9 years) and most commonly involved the parotid glands, with frequent extraglandular extension. Of all 17 cases reported in the literature, $61.5 \%$ were female versus $38.5 \%$ males. They appeared more aggressive than conventional EMC, mainly due to high propensity for lymph node and distant metastasis, prominent infiltrative growth pattern, and higher proliferative activity. However, little follow-up information is available, with a mean of 27.6 months (range: 3 to 72 months). The worse prognosis suggests the need for wider excision, neck dissection, and adjuvant radiotherapy $[5,10,45-50]$.

Five cases of polymorphous low-grade adenocarcinoma (PLGA) with transformation to high-grade carcinoma described in the literature showed significant similarities in the morphology and in the origin, minor salivary gland of palate. These cases underwent transformation to poorly differentiated adenocarcinomas characterized by a predominantly solid and cystic growth pattern, nuclear atypia with prominent nucleoli and foci of necrosis $[18,51,52]$ Lloreta et al. described one more case originating in the nasal cavity and adjacent sinuses with extensive areas of undifferentiated carcinoma consisting of compact epithelial cell nests with central necrosis [53]. The histological transformation seems to have occurred after a protracted clinical course with multiple recurrences, a late phenomenon in tumor progression $[18,52]$. The possible role of radiation therapy as an initiator of this transformation may have been important in the three of the five published cases of transformation in PLGA $[51,52]$. Despite the fact that high-grade transformation is recognized as an event with a more aggressive clinical course, only one case of PLGA in the literature died in consequence of the disease [53] and none showed metastasis thereafter $[18,51,52]$.

The only two cases reported of dedifferentiated mucoepidermoid carcinoma (MEC) in the literature both presented a biphasic histology comprising a high-grade component and a low grade component separated by a transition zone $[3,54]$. The high-grade component exhibited solid nests and sheetlike growth patterns, without glandular or cystic structures. Sheets of undifferentiated anaplastic or sarcomatoid growth with marked pleomorphism, frequent mitoses $(>50 \%)$, and extensive necrosis were also observed (Figure 3) [3, 54].

The first case described by Nagao et al. concerned a 55 -year-old man with a parotid gland tumor. Despite two recurrences within a short period after surgery, the patient remained alive during the next 10 years [3]. The second case of an 11-year-old girl located in trachea, conversely, rapidly metastasized to pleura, mediastinal lymph nodes, abdominal wall and vertebral bones leading to death in $<3$ months from diagnosis [54]. With few cases in the literature, it is difficult to establish clinical correlations in relation to the conventional MEC, but dedifferentiated MEC seems to reach the same broad age range as the conventional counterpart.

One case of dedifferentiated myoepithelial carcinoma (MCa) was described by Ogawa et al. in 2003 [4]. Two histologically distinct neoplastic cell populations were observed in the multinodular tumor of parotid gland. The first population was diagnosed as low-grade $\mathrm{MCa}$ and occupied more than $80 \%$ of the tumor. The second population consisted of polygonal or short spindle cells with pleomorphism as well as infiltration and high mitotic rate suggesting undifferentiated carcinoma. Moreover, these tumor cells lost the immunohistochemical characteristics of myoepithelial differentiation. A 59-year-old man presented first recurrence in the primary site after 5 months and a second one was observed 4 months later, although radiation therapy was used. The patient is alive and metastasis was not recognized [4].

\section{Immunohistochemical Profile}

3.1. Myoepithelial Markers. Myoepithelial cells exhibit dual epithelial and smooth muscle characteristics and traditionally are stained with antibodies against myoid proteins, such as $\alpha$-smooth muscle actin ( $\alpha$-SMA), muscle-specific actin (HHF35), vimentin, or calponin. Recently, p63 has become a popular marker for abluminal cells (basal cells and myoepithelial cells) [55-57]. In tumors with myoepithelial component, such as AdCC and EMC, the participation of myoepithelial cells in the dedifferentiated/high-grade transformed areas seems to differ markedly. In AdCC, the loss of myoepithelial component has been used as one of the major criteria to identify the transformed areas [8]. Thus the expression of p63, $\alpha$-SMA, and calponin should be absent or at least focal in the high-grade component [8]. In contrast, in EMC the myoepithelial participation appears to be important in the high-grade component [10], although the cells show a heterogeneous expression of the myoepithelial markers (Table 1). S-100 protein and p63 have been found to be diffusely or focally positive in the transformed component in many dedifferentiated/highgrade transformed EMC, whereas $\alpha$-SMA was rarely detected and calponin negative $[5,10,45,46,50]$. It should be emphasized that neoplastic myoepithelial cells present a great plasticity in terms of immunoprofile and in EMC, even the conventional tumors show differences regarding the expression of myoepithelial markers [48]. In these, p63 expression is more frequently encountered than SMA.

3.2. Other Markers. The immunoprofile of the dedifferentiation/high-grade transformation in salivary gland tumors has been evaluated in many studies (Table 1), however, with variable and therefore inconclusive results, possibly due to the small number of cases. The marker that best distinguished between the transformed and the conventional components was $\mathrm{Ki}-67$, since, in all salivary gland tumors studied, an increased proliferation index was detected in the transformed component when compared to the conventional counterpart $[2,3,5,8,9,11,12,18,21,22,25,27,28,36,45$, $48,49,54]$. However, a distinct cut-off for the proliferation index that could identify the transformed component has 


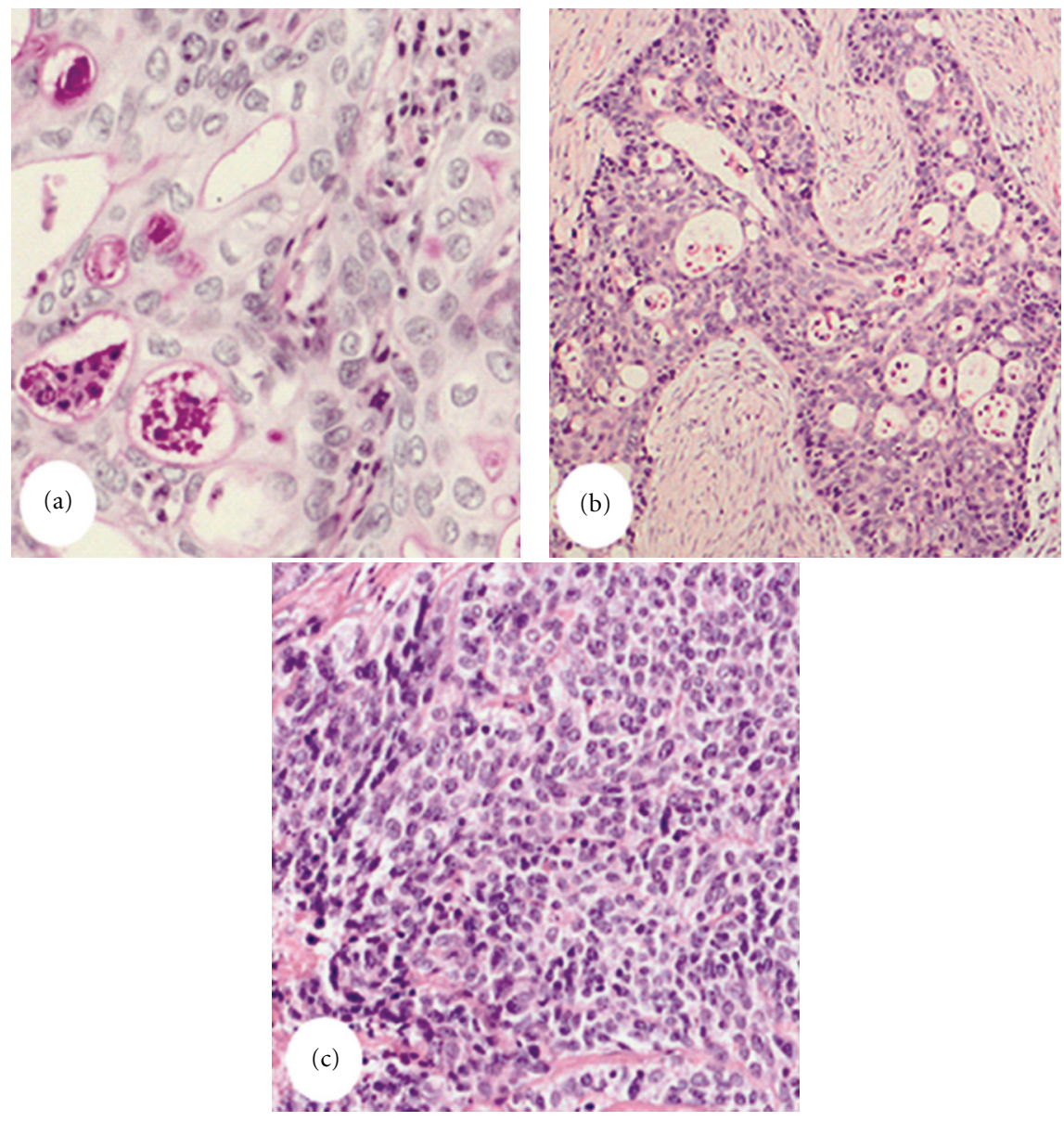

FIGURE 3: Mucoepidermoid carcinoma with dedifferentiation to undifferentiated carcinoma. (a) and (b), Conventional component; (c) dedifferentiated component. (a) PAS original magnification 400x; (b) H\&E original magnification 100x; (c) H\&E original magnification 200x.

yet to be established and probably it is variable among the different types of tumors. The expression of p53 in the transformed areas showed, in most of the cases and in most of the salivary tumor types, higher levels than in the conventional areas. Nevertheless, Di Palma et al. (1999) and Henley et al. (1997) showed negative expression of this protein in the dedifferentiated/high-grade component of AcCC [35, 36]. Fonseca et al. and Sarode et al. also showed lack of specificity to p53 in both components of their cases of EMC [49, 50]. These inconsistent results suggest that TP53 alteration is not the only mechanism for transformation in salivary gland tumors but may indicate a poor prognosis, similar to what is known for conventional AdCC [58]. Cyclin D1 is an important regulator of the G1 phase of cell cycle [59]. Positive expression was observed in dedifferentiated/transformed and conventional area of AdCC, AcCC, and EMC [5, 8, 9, 21, 25, 28]. However, in the MEC, Subramaniam et al. did not find positive expression in the dedifferentiated area [54]. The precise mechanisms responsible for the observed cyclin D1 overexpression in dedifferentiated/high-grade transformed salivary gland tumors (Table 1) are not fully established; a role in dedifferentiation of AdCC has been suggested in early studies
$[2,21]$. Gene amplification of cyclin D1 might contribute, as has been described in conventional AdCC [60].

Many other markers such as b-catenin [9], E-cadherin [27], pRB [28], BCL2 [18], and glucose transporters (GLUT) have been studied in individual tumors and deserve to be analyzed in other salivary gland tumors with dedifferentiation/high-grade transformation. GLUT1 has been considered a key molecule regulating the transport and metabolism of glucose. Overexpression of GLUT1 has been correlated with poor prognosis, tumor aggressiveness, and lymph node metastases [61]. Bonfitto et al. showed increased expression of GLUT1 in the transformed area when compared to conventional area of AdCC, suggesting a change in metabolic state of cancer cells imposing an increased utilization of energy. However, the authors did not find any correlation between GLUT1 expression and clinical outcome [11]. In summary, the immunohistochemical differences between the conventional and transformed areas require further studies.

\section{Molecular Profile}

Studies on the genetic changes in salivary gland tumors with dedifferentiation/high-grade transformation are rare. Only 
TABLE 1: Immunoprofile of dedifferentiated/high-grade transformed areas in salivary gland tumors.

\begin{tabular}{|c|c|c|c|c|c|c|}
\hline \multirow{2}{*}{ Antibodies } & \multicolumn{6}{|c|}{ Immunoprofile of high-grade transformed areas } \\
\hline & AdCC & AcCC & EMC & PLGA & MEC & $\mathrm{MCa}$ \\
\hline \multicolumn{7}{|l|}{ Proliferative antigen } \\
\hline Ki-67 & $\mathrm{HI}$ & $\mathrm{HI}$ & $\mathrm{HI}$ & $\mathrm{HI}$ & $\mathrm{HI}$ & $\mathrm{HI}$ \\
\hline \multicolumn{7}{|l|}{ Cytokeratins } \\
\hline AE1-AE3 & + & $+/ F$ & + & + & $+/-$ & $F$ \\
\hline CAM 5.2 & + & NA & $+/ F$ & + & - & NA \\
\hline $34 \mathrm{bE} 12$ & + & NA & $F$ & NA & NA & NA \\
\hline CK 5/6 & NA & - & + & NA & NA & NA \\
\hline CK 7 & NA & - & $+/-$ & + & NA & NA \\
\hline CK 14 & $-/ F$ & - & $-/ F$ & NA & NA & NA \\
\hline CK 20 & NA & - & NA & - & $\mathrm{NA}$ & NA \\
\hline \multicolumn{7}{|l|}{ Myoepithelial cell } \\
\hline S-100 & $+/ F /-$ & $+/-$ & $+/ F /-$ & $+/-$ & - & - \\
\hline $\begin{array}{l}\alpha \text {-SMA (alfa-smooth } \\
\text { muscle actin) }\end{array}$ & $-/ F$ & - & $-/ F$ & - & - & - \\
\hline p63 - n & $-/ F$ & NA & $+/ F /-$ & $+/-$ & NA & NA \\
\hline Vimentin & NA & + & $+/-$ & $+/-$ & $\mathrm{NA}$ & + \\
\hline Calponin & $F$ & - & - & $-/ F$ & NA & NA \\
\hline $\begin{array}{l}\text { HHF35 (muscle-specific } \\
\text { actin) }\end{array}$ & $-/ F$ & NA & - & NA & NA & NA \\
\hline Desmin & NA & NA & - & - & NA & NA \\
\hline \multicolumn{7}{|l|}{ Cell cycle control } \\
\hline p53 & $\mathrm{HI} /+$ & $\mathrm{HI} /-$ & $+/-$ & $\mathrm{HI}$ & $\mathrm{HI} / F$ & + \\
\hline Cyclin D1 & $+/ F$ & HI & + & NA & NA & + \\
\hline \multicolumn{7}{|l|}{ Membrane receptors } \\
\hline C-kit (CD117) & $+/-$ & - & NA & NA & - & NA \\
\hline HER2/neu (c-erbB2) & $+/-$ & + & - & + & - & NA \\
\hline $\begin{array}{l}\text { EMA (epithelial membrane } \\
\text { antigen) }\end{array}$ & + & NA & + & + & + & NA \\
\hline \multicolumn{7}{|l|}{ Structural proteins } \\
\hline $\begin{array}{l}\text { GFAP (glial fibrillary acidic } \\
\text { protein) }\end{array}$ & $-/ F$ & NA & - & $+/-$ & - & NA \\
\hline \multicolumn{7}{|l|}{ Cell Adhesion proteins } \\
\hline $\begin{array}{l}\text { CEA (carcinoembryonic } \\
\text { antigen) }\end{array}$ & $-/ F$ & NA & + & $+/-$ & - & NA \\
\hline \multicolumn{7}{|l|}{ Steroid receptor } \\
\hline Androgen receptor & - & - & NA & $+/ F$ & NA & NA \\
\hline
\end{tabular}

+: positive expression; -: negative expression; F: focal expression; NA: not available; HI: higher index than in conventional area; AdCC: adenoid cystic carcinoma; AcCC: acinic cell carcinoma; EMC: epithelial-myoepithelial carcinoma; PLGA: polymorphous low-grade adenocarcinoma; MEC: mucoepidermoid carcinoma; MCa: myoepithelial carcinoma.

few cases have been demonstrated to carry mutations or deletions in TP53 gene by loss of heterozygosity (LOH), polymerase chain reaction (PCR), and microarray comparative genomic hybridization (CGH) $[9,12,21,28,35,36]$. However, p53 positive immunostaining is often indicative of mutations in TP53 and was observed in the transformed component in all tumor types studied $[2-5,8,9,11,12$,
$18,21,22,25,27,28,54]$. Therefore, the evidence suggests that p53 abnormalities may be implicated in the process of dedifferentiation, although its real importance in this process should be clarified by further molecularstudies.

Fluorescence in situ hybridization (FISH) analysis did not demonstrate gene amplification in the transformed area with mild overexpression of HER-2/neu protein in 
acinic cell carcinoma [9]. DNA content has been studied only in dedifferentiated/high-grade transformed AcCC and EMC. Aneuploid AcCCs were associated with poor clinical outcome whereas no aneuploid tumor was found in the EMC group $[31,35,36]$.

Using a high-resolution microarray CGH analysis in AdCC with high-grade transformation, a correlation between the number of chromosomal aberrations and the degree of gland differentiation of the transformed component was found. The AdCC with transformation in moderately differentiated carcinomas carried one single abnormality, whereas the AdCC with transformation in poorly differentiated carcinomas showed a higher number of alterations. These findings suggest that the AdCC with high-grade transformation may not necessarily reflect a more advanced stage of tumor progression, but rather a transformation to another histological form, which encompasses a wide spectrum of carcinomas in terms of aggressiveness [12]. A comparison of the microarray CGH results of the transformed and the conventional components in two cases of AdCC with high-grade transformation (unpublished data on cases in [12]) showed identical genetic profiles. A search in the literature shows that this finding is not unusual. Among others, examples are dedifferentiated liposarcomas and biphasic carcinosarcomas $[62,63]$. This indicates that the genetic abnormalities have been acquired early in tumorigenesis, or at least before the start of the phenotypic change. It may be speculated that the genetic changes not detectable by microarray CGH analysis such as mutation or epigenetic silencing underlie the phenotypic change.

In conclusion, the dedifferentiation/high-grade transformation in salivary gland tumors seems to be a more complex process than simple progression through histological grades.

Currently, the most useful tool in identifying the transformed component is still a combination of morphological criteria aided by Ki67 expression analysis.

\section{Acknowledgments}

The paper is supported in Brazil by Fundação de Amparo à Pesquisa do Estado de São Paulo (FAPESP), Grant no. 2009/ 54377-2 and 2010/51571-0, and in Spain by EMER07-048 of Fondos de Investigación Sanitaria (FIS) and RD06/0020/ 0034 of Red Temática de Investigación Cooperativa en Cáncer (RTICC), Spain.

\section{References}

[1] D. C. Dahlin and J. W. Beabout, "Dedifferentiation of lowgrade chondrosarcomas," Cancer, vol. 28, no. 2, pp. 461-466, 1971.

[2] W. Cheuk, J. K. Chan, and R. K. Ngan, "Dedifferentiation in adenoid cystic carcinoma of salivary gland: an uncommon complication associated with an accelerated clinical course," The American Journal of Surgical Pathology, vol. 23, no. 4, pp. 465-472, 1999.

[3] T. Nagao, T. A. Gaffey, P. A. Kay et al., "Dedifferentiation in low-grade mucoepidermoid carcinoma of the parotid gland," Human Pathology, vol. 34, no. 10, pp. 1068-1072, 2003.
[4] I. Ogawa, T. Nishida, M. Miyauchi, S. Sato, and T. Takata, "Dedifferentiated malignant myoepithelioma of the parotid gland," Pathology International, vol. 53, no. 10, pp. 704-709, 2003.

[5] K. Kusafuka, Y. Takizawa, T. Ueno et al., "Dedifferentiated epithelial-myoepithelial carcinoma of the parotid gland: a rare case report of immunohistochemical analysis and review of the literature," Oral Surgery, Oral Medicine, Oral Pathology, Oral Radiology and Endodontology, vol. 106, no. 1, pp. 909915, 2008.

[6] R. J. Stanley, L. H. Weiland, K. D. Olsen, and B. W. Pearson, "Dedifferentiated acinic cell (acinous) carcinoma of the parotid gland," Otolaryngology_Head and Neck Surgery, vol. 98, no. 2, pp. 155-161, 1988.

[7] M. Katoh, C. Shaw, Q. Xu et al., "An orderly retreat: dedifferentiation is a regulated process," Proceedings of the National Academy of Sciences of the United States of America, vol. 101, no. 18, pp. 7005-7010, 2004.

[8] R. R. Seethala, J. L. Hunt, Z. W. Baloch, V. A. LiVolsi, and E. Leon Barnes, "Adenoid cystic carcinoma with high-grade transformation: a report of 11 cases and a review of the literature," The American Journal of Surgical Pathology, vol. 31, no. 11, pp. 1683-1694, 2007.

[9] A. Skálová, R. Sima, T. Vanecek et al., "Acinic cell carcinoma with high-grade transformation: a report of 9 cases with immunohistochemical study and analysis of TP53 and HER2/neu genes," The American Journal of Surgical Pathology, vol. 33, no. 8, pp. 1137-1145, 2009.

[10] P. Roy, M. J. Bullock, B. Perez-Ordoñez, I. Dardick, and I. Weinreb, "Epithelial-myoepithelial carcinoma with high grade transformation," The American Journal of Surgical Pathology, vol. 34, no. 9, pp. 1258-1265, 2010.

[11] V. L. Bonfitto, A. P. Demasi, A. F. Costa, J. F. Bonfitto, V. C. Araujo, and A. Altemani, "High-grade transformation of adenoid cystic carcinomas: a study of the expression of GLUT1 glucose transporter and of mitochondrial antigen," Journal of Clinical Pathology, vol. 63, no. 7, pp. 615-619, 2010.

[12] A. F. Costa, A. Altemani, H. Vécony et al., "Genetic profile of adenoid cystic carcinomas (ACC) with high-grade transformation versus solid type," Analytical Cellular Pathology, vol. 33 , no. 5, pp. 217-228, 2010.

[13] P. C. Nowell, "The clonal evolution of tumor populations," Science, vol. 194, pp. 23-28, 1976.

[14] N. E. Navin and J. Hicks, "Tracing the tumor lineage," Molecular Oncology, vol. 4, pp. 267-283, 2010.

[15] L. Torres, F. R. Ribeiro, N. Pandis, J. A. Andersen, S. Heim, and M. R. Teixeira, "Intratumor genomic heterogeneity in breast cancer with clonal divergence between primary carcinomas and lymph node metastases," Breast Cancer Research and Treatment, vol. 102, no. 2, pp. 143-155, 2007.

[16] N. Navin, A. Krasnitz, L. Rodgers et al., "Inferring tumor progression from genomic heterogeneity," Genome Research, vol. 20, no. 1, pp. 68-80, 2010.

[17] Y. Kanai, "Alterations of DNA methylation and clinicopathological diversity of human cancers," Pathology International, vol. 58, no. 9, pp. 544-558, 2008.

[18] R. H. Simpson, J. S. Reis-Filho, E. M. Pereira, A. C. Ribeiro, and A. Abdulkadir, "Polymorphous low-grade adenocarcinoma of the salivary glands with transformation to high-grade carcinoma," Histopathology, vol. 41, no. 3, pp. 250-259, 2002.

[19] A. K. El-Naggar and A. G. Huvos, "Adenoid cystic carcinoma," in World Health Organization Classification of Head and Neck Tumours, L. Barnes, J. W. Evenson, P. Reichart et al., Eds., pp. 221-222, IARC Press, Lyon, France, 2005. 
[20] M. A. Moles, I. R. Avila, and A. R. Archila, "Dedifferentiation occurring in adenoid cystic carcinoma of the tongue," Oral Surgery, Oral Medicine, Oral Pathology, Oral Radiology and Endodontology, vol. 88, pp. 177-180, 1999.

[21] Y. P. Chau, T. Hongyo, K. Aozasa, and J. K. Chan, "Dedifferentiation of adenoid cystic carcinoma: report of a case implicating p53 gene mutation," Human Pathology, vol. 32, no. 12, pp. 1403-1407, 2001.

[22] F. Ide, K. Mishima, and I. Saito, "Small foci of high-grade carcinoma cells in adenoid cystic carcinoma represent an incipient phase of dedifferentiation," Histopathology, vol. 43, no. 6, pp. 604-606, 2003.

[23] S. Brackrock, A. Krüll, K. Röser, R. Schwarz, L. Riethdorf, and W. Alberti, "Neutron therapy, prognostic factors and dedifferentiation of adenoid cystic carcinomas (ACC) of salivary glands," Anticancer Research, vol. 25, no. 2, pp. 13211326, 2005.

[24] Y. Hayashido, H. Yoshioka, N. Tanaka et al., "Dedifferentiation in adenoid cystic carcinoma of submaxillary gland: a case of report,” Oral Oncology Extra, vol. 41, no. 5, pp. 84-88, 2005.

[25] K. Sato, Y. Ueda, A. Sakurai et al., "Adenoid cystic carcinoma of the maxillary sinus with gradual histologic transformation to high-grade adenocarcinoma: a comparative report with dedifferentiated carcinoma," Virchows Archiv, vol. 448, no. 2, pp. 204-208, 2006.

[26] A. Handra-Luca, D. Planchard, and P. Fouret, "Docetaxelcisplatin-radiotherapy in adenoid cystic carcinoma with highgrade transformation," Oral Oncology, vol. 45, no. 11, pp. e208-e209, 2009.

[27] K. P. Malhotra, V. Agrawal, and R. Pandey, "High grade transformation in adenoid cystic carcinoma of the parotid: report of a case with cytologic, histologic and immunohistochemical study," Head and Neck Pathology, vol. 3, no. 4, pp. 310-314, 2009.

[28] T. Nagao, T. A. Gaffey, H. Serizawa et al., "Dedifferentiated adenoid cystic carcinoma: a clinicopathologic study of 6 cases," Modern Pathology, vol. 16, no. 12, pp. 1265-1272, 2003.

[29] G. L. Ellis and R. L. Corio, "Acinic cell adenocarcinoma. A clinicopathologic analysis of 294 cases," Cancer, vol. 52, no. 3, pp. 542-549, 1983.

[30] J. G. Batsakis, M. A. Luna, and A. K. El-Naggar, "Histopathologic grading of salivary gland neoplasms:acinic cell carcinomas," Annals of Otology, Rhinology and Laryngology, vol. 99, pp. 929-933, 1990.

[31] A. K. El-Naggar, J. G. Batsakis, M. A. Luna, D. McLemore, and R. M. Byers, "DNA flow cytometry of acinic cell carcinomas of major salivary glands," Journal of Laryngology and Otology, vol. 104, no. 5, pp. 410-416, 1990.

[32] C. Colmenero, M. Patron, and I. Sierra, "Acinic cell carcinoma of the salivary glands. A review of 20 new cases," Journal of Cranio-Maxillo-Facial Surgery, vol. 19, no. 6, pp. 260-266, 1991.

[33] J. F. Nunes, I. Fonseca, and J. Soares, "Helioid inclusions in dedifferentiated acinic cell carcinoma of the parotid gland," Ultrastructural Pathology, vol. 20, no. 5, pp. 443-449, 1996.

[34] G. L. Ellis and P. L. Auclair, Tumors of the Salivary Glands, Atlas of Tumor Pathology, Armed Forces Institute of Pathology, Washington, DC, USA, 1996.

[35] J. D. Henley, W. A. Geary, C. L. Jackson, C. D. Wu, and D. R. Gnepp, "Dedifferentiated acinic cell carcinoma of the parotid gland: a distinct rarely described entity," Human Pathology, vol. 28, no. 7, pp. 869-873, 1997.

[36] S. Di Palma, V. Corletto, C. Lavarino, S. Birindelli, and S. Pilotti, "Unilateral aneuploid dedifferentiated acinic cell carcinoma associated with bilateral-low grade diploid acinic cell carcinoma of the parotid gland," Virchows Archiv, vol. 434, no. 4, pp. 361-365, 1999.

[37] D. R. Johnson, M. C. N. Perez, F. K. Baksh et al., "Acinic cell carcinoma and dedifferentiated acinic cell carcinoma of the parotid gland," Modern Pathology, vol. 13, p. 139A, 2000, (abstract no. 810).

[38] S. Piana, A. Cavazza, C. Pedroni, R. Scotti, L. Serra, and G. Gardini, "Dedifferentiated acinic cell carcinoma of the parotid gland with myoepithelial features," Archives of Pathology and Laboratory Medicine, vol. 126, no. 9, pp. 1104-1105, 2002.

[39] M. Prasad and D. H. Kraus, "Acinic cell carcinoma of the parotid gland presenting as an external auditory canal mass," Head and Neck, vol. 26, no. 1, pp. 85-88, 2004.

[40] P. González-Peramato, J. A. Jiménez-Heffernan, P. LópezFerrer, B. Vicandi, and J. M. Viguer, "Fine neeedle aspiration cytology of dedifferentiated acinic cell carcinoma of the parotid gland: a case report," Acta Cytologica, vol. 50, no. 1, pp. 105-108, 2006.

[41] S. Johnykutty, C. H. Miller, R. S. Hoda, and E. J. Giampoli, "Fine-needle aspiration of dedifferentiated acinic cell carcinoma: report of a case with cyto-histological correlation," Diagnostic Cytopathology, vol. 37, no. 10, pp. 763-768, 2009.

[42] J. G. Batsakis, E. K. Chinn, T. A. Weimert, W. P. Work, and C. J. Krause, "Acinic cell carcinoma: a clinicopathologic study of thirty-five cases," Journal of Laryngology and Otology, vol. 93, no. 4, pp. 325-340, 1979.

[43] O. J. Hyun, I. R. Yoo, C. K. Jung, S. H. Kim, and S. K. Chung, "F-18 FDG PET/CT findings of dedifferentiated acinic cell carcinoma," Clinical Nuclear Medicine, vol. 35, no. 6, pp. 473474,2010 .

[44] I. Fonseca and J. Soares, "Epithelial-myoepithelial carcinoma," in World Health Organization Classification of Head and Neck Tumours, L. Barnes, J. W. Evenson, P. Reichart et al., Eds., pp. 225-226, IARC Press, Lyon, France, 2005.

[45] L. Alós, R. Carrillo, J. Ramos et al., "High-grade carcinoma component in epithelial-myoepithelial carcinoma of salivary glands clinicopathological, immunohistochemical and flowcytometric study of three cases," Virchows Archiv, vol. 434, no. 4, pp. 291-299, 1999.

[46] M. Niederhagen, P. Zengel, and S. Ihrler, "Secondary highmalignant transformation of a low-malignant epithelialmyoepithelial carcinoma," Pathologe, vol. 30, no. 6, pp. 461465, 2009.

[47] R. H. Simpson, T. J. Clarke, P. T. Sarsfield, and P. G. Gluckman, "Epithelial-myoepithelial carcinoma of salivary glands," Journal of Clinical Pathology, vol. 44, no. 5, pp. 419423, 1991.

[48] R. R. Seethala, E. L. Barnes, and J. L. Hunt, "Epithelialmyoepithelial carcinoma: a review of the clinicopathologic spectrum and immunophenotypic characteristics in 61 tumors of the salivary glands and upper aerodigestive tract," The American Journal of Surgical Pathology, vol. 31, no. 1, pp. 44-57, 2007.

[49] I. Fonseca, A. Felix, and J. Soares, "Dedifferentiation in salivary gland carcinomas," The American Journal of Surgical Pathology, vol. 24, no. 3, pp. 469-471, 2000.

[50] V. R. Sarode, J. Truelson, and M. Zaidie, "Dedifferentiated epithelial-myoepithelial carcinoma of the parotid gland with aberrant expression of prostate specific antigen: a case report," International Journal of Surgical Pathology, vol. 18, no. 5, pp. 401-405, 2010.

[51] S. E. Mills, T. A. Garland, and M. S. Allen Jr., "Low-grade papillary adenocarcinoma of palatal salivary gland origin," The 
American Journal of Surgical Pathology, vol. 8, no. 5, pp. 367374, 1984.

[52] T. J. Pelkey and S. E. Mills, "Histologic transformation of polymorphous low-grade adenocarcinoma of salivary gland," The American Journal of Clinical Pathology, vol. 111, no. 6, pp. 785-791, 1999.

[53] J. Lloreta, S. Serrano, J. M. Corominas, E. Ferres-Padro, and I. Dardick, "Polymorphous low-grade adenocarcinoma arising in the nasal cavities with an associated undifferentiated carcinoma," Ultrastructural Pathology, vol. 19, no. 5, pp. 365370, 1995.

[54] M. M. Subramaniam, S. B. Ng, S. B. Seah, D. Anuar, R. Soong, and V. K. Lee, "Molecular characterization of dedifferentiated mucoepidermoid carcinoma of the trachea using laser microdissection-based TP53 mutation analysis," Histopathology, vol. 55, no. 4, pp. 472-475, 2009.

[55] Y. Ogawa, "Immunocytochemistry of myoepithelial cells in the salivary glands," Progress in Histochemistry and Cytochemistry, vol. 38, no. 4, pp. 343-426, 2003.

[56] H. Bilal, A. Handra-Luca, J. C. Bertrand, and P. J. Fouret, "P63 is expressed in basal and myoepithelial cells of human normal and tumor salivary gland tissues," Journal of Histochemistry and Cytochemistry, vol. 51, no. 2, pp. 133-139, 2003.

[57] P. C. Edwards, T. Bhuiya, and R. D. Kelsch, "Assessment of p63 expression in the salivary gland neoplasms adenoid cystic carcinoma, polymorphous low-grade adenocarcinoma, and basal cell and canalicular adenomas," Oral Surgery, Oral Medicine, Oral Pathology, Oral Radiology and Endodontics, vol. 97, no. 5, pp. 613-619, 2004.

[58] D. E. da Cruz Perez, F. de Abreu Alves, I. Nobuko Nishimoto, O. P. de Almeida, and L. P. Kowalski, "Prognostic factors in head and neck adenoid cystic carcinoma," Oral Oncology, vol. 42, no. 2, pp. 139-146, 2006.

[59] R. Donnellan and R. Chetty, "Cyclin D1 and human neoplasia," Molecular Pathology, vol. 51, no. 1, pp. 1-7, 1998.

[60] G. Sequeiros-Santiago, D. García-Carracedo, M. F. Fresno, C. Suarez, J. P. Rodrigo, and M. V. Gonzales, "Oncogene amplification pattern in adenoid cystic carcinoma of the salivary glands," Oncology Reports, vol. 21, no. 5, pp. 12151222, 2009.

[61] T. Kawamura, T. Kusakabe, T. Sugino et al., "Expression of glucose transporter-1 in human gastric carcinoma: association with tumor aggressiveness, metastasis, and patient survival," Cancer, vol. 92, no. 3, pp. 634-641, 2001.

[62] H. Vékony, C. R. Leemans, B. Ylstra, G. A. Meijer, I. van der Waal, and E. Bloemena, "Salivary gland carcinosarcoma: oligonucleotide array CGH reveals similar genomic profiles in epithelial and mesenchymal components," Oral Oncology, vol. 45, no. 3, pp. 259-265, 2009.

[63] A. E. Horvai, S. De Vries, R. Roy, R. J. O’Donnell, and F. Waldman, "Similarity in genetic alterations between paired well-differentiated and dedifferentiated components of dedifferentiated liposarcoma," Modern Pathology, vol. 22, no. 11, pp. 1477-1488, 2009. 


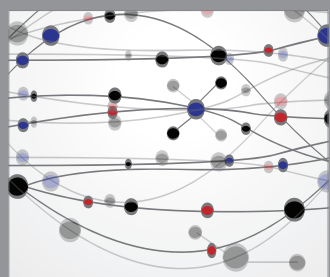

The Scientific World Journal
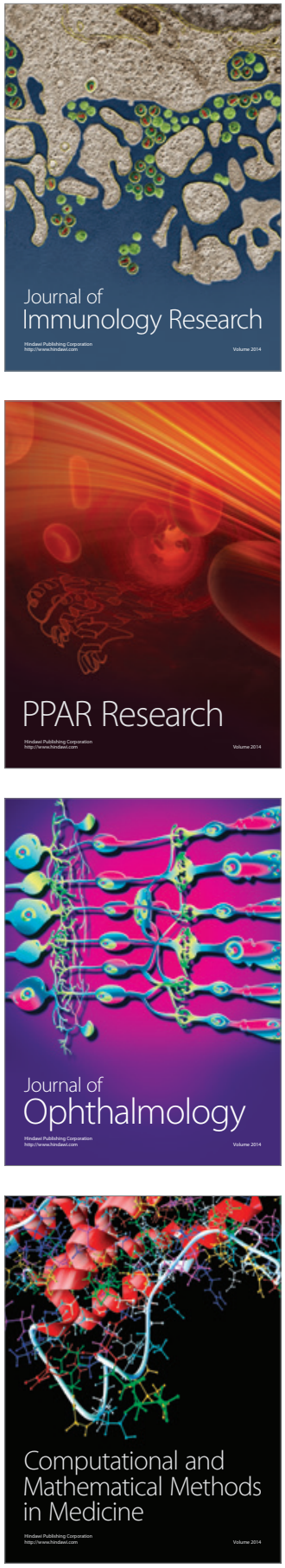

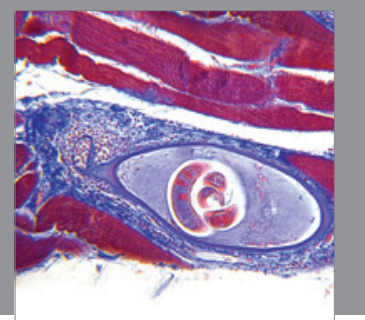

Gastroenterology

Research and Practice
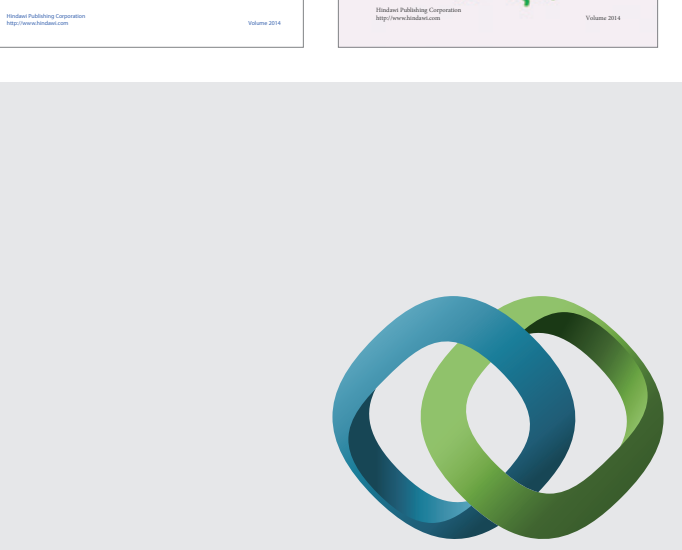

\section{Hindawi}

Submit your manuscripts at

http://www.hindawi.com
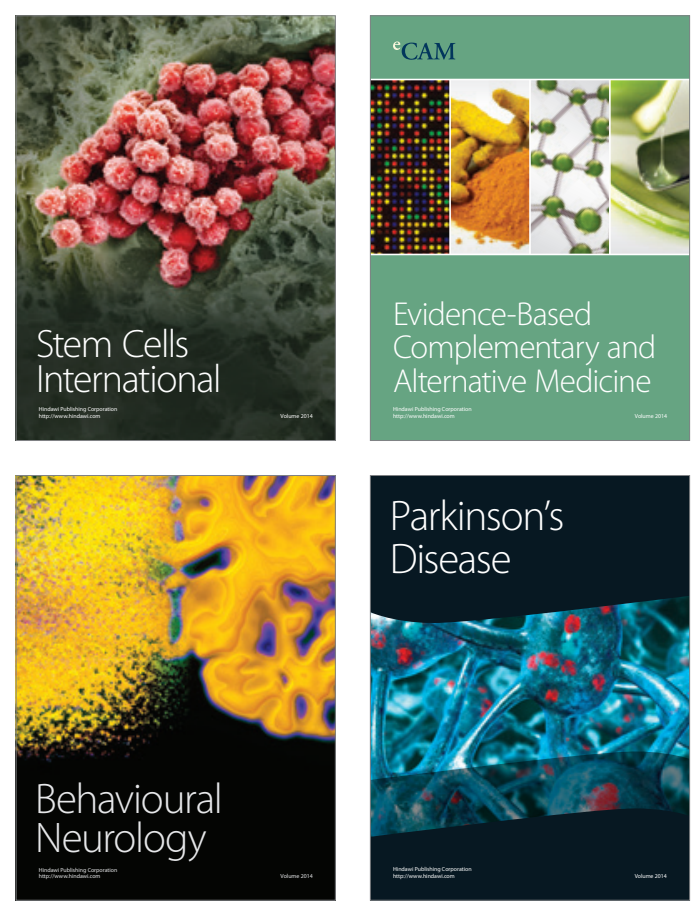

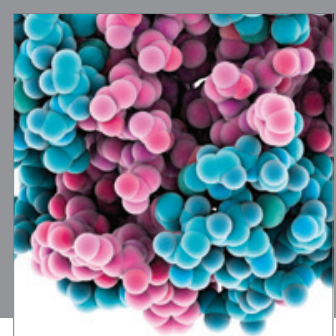

Journal of
Diabetes Research

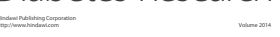

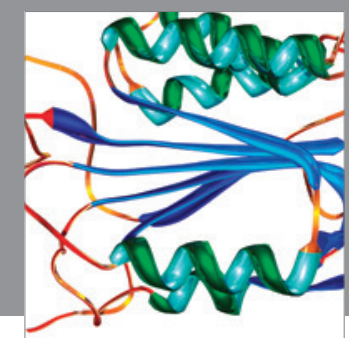

Disease Markers
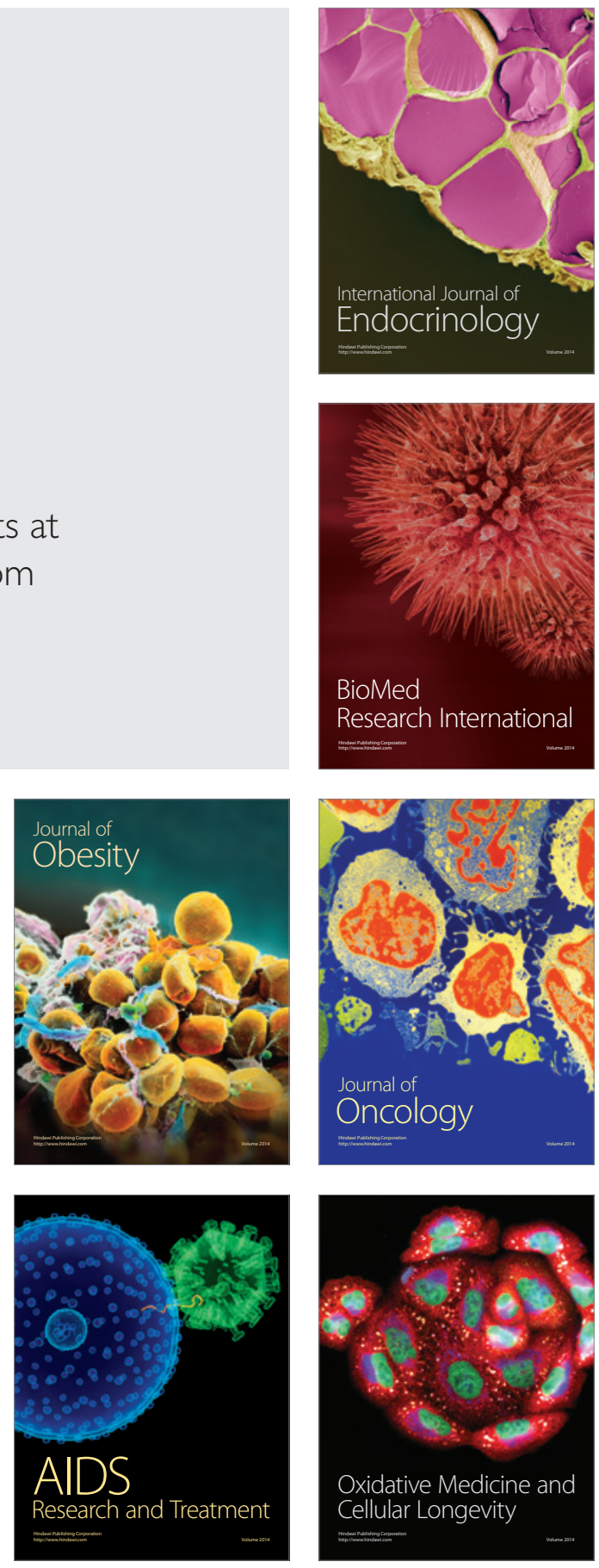